\title{
SUPER HEDGING BY DUALITY IN PRESENCE OF SEMIMARTINGALE ASSETS
}

\author{
Luca Decarli \\ Department of Mathematics \\ University of Trento \\ via Sommarive, 14-38123 Povo (Trento), ITALY
}

\begin{abstract}
The superhedging problem in a free-arbitrage, incomplete market, is classically solved by mean of the Skorokhod Embedding Problem (SEP) approach, but only in the case of derivatives with time invariant payoff. To avoid such a restriction, we exploit the MongeKantorovich (MK) dual approach that turns the starting problem into a problem of stochastic optimization with linear constrain and does not require any time invariant property, in particular the superhedging dual problem for a derivative $\xi$, turns to be its lower bound fair- price for the market. The paper is focused on generalizing the hypothesis on the dynamics of the underlying assets characterizing a given financial market. In particular we extend the results provided by Galichon, Labordere and Touzi in [12], to consider assets whose dynamic admits continuous semimartingale properties. We also provide an alternative dual formulation of the initial problem which allows to apply numerical approximation schemes to the case of super hedging a derivative.
\end{abstract}

AMS Subject Classification: 47N10, 60G40, 60G48, 60H15, 60H35, 62L15, 90-08, 91G20, 91G80

Key Words: stochastic optimization, Skorokhod embedding theorem, optimal transportation theory, stochastic hedging problems, stopping times, Monte Carlo simulations

\section{Introduction}

In the present paper we solve the superhedging problem (SP), when the involved assets are allowed to be semimartingales. Our approach is based on the theory

Received: August 2, 2016

Published: September 10, 2016 (c) 2016 Academic Publications, Ltd. url: www.acadpubl.eu 
of stochastic optimal transport and related duality results.

In the original Monge-Kantorovich (MK) mass transportation problem, we have two probability distributions on $\mathbb{R}^{d}$, namely the initial one $\mu_{0}$ and the terminal one $\mu$. An admissible transportation plan is defined as a stochastic processes $X:=\left\{X_{t}\right\}_{t \in[0, T]}$, such that the marginal distributions associated to the random vector $\left(X_{0}, X_{T}\right)$ are $\mu_{0}$ and $\mu$.

For such a stochastic process $X$, the cost needed to transport a mass from the distribution $\mu_{0}$ to the distribution $\mu$, also called plan transportation cost, or equivalently from the position $X_{0}(\omega)$ to the position $X_{T}(\omega)$, is given by $\mathbb{E}\left[c\left(X_{0}, X_{1}\right)\right]$, provided the specification of some cost function $c: \mathbb{R}^{d} \times \mathbb{R}^{d} \longmapsto$ $\mathbb{R}_{+}$. Then the optimization task consists in minimizing the aforementioned cost among all admissible transportation plans.

Focusing our attention on the financial counterpart of the general setting already described, the latter turn out to be the quest for solving the problem of superhedging, for certain type of financial quantities to better specify later. In particular, within the financial framework formulation we are interested in, one has $\mu_{0}=\delta_{X_{0}}$, where $\delta_{X_{0}}$ represents the distribution whose mass is concentrated in $X_{0}$ which constitutes the initial capital $X_{0} \in \mathbb{R}_{+}$, of the investment we are interested in. In what follows we denote by $S$ the underlying, possibly multidimensional, risky asset, also supposing the existence of more than one Equivalent Martingale Measure $(\mathrm{EMM}) \mathbb{Q}$, which implies that we will deal with an incomplete market model which implies that there exists at least one elementary security which is not attainable. We recall that the existence of an EMM is equivalent to require the absence of arbitrage opportunities, while, if there exists a unique EMM, then the Law of a unique fair price holds.

It is worth to mention that the dual formulation of the (SP) turns out to be the quest for the upper bound price associated to a derivative $\xi$ stated on the aforementioned asset $S$. In particular, let us model our market w.r.t. a probability space $\left(\Omega, \mathbb{F}, \mathcal{F}_{t \geq 0}, \mathbb{P}\right)$, and we fix a finite time maturity $T$, supposing that $\mathcal{F}_{T}=\mathbb{F}$, then the no-arbitrage assumption assures that any pathdependent security derivative $\xi\left(S_{t}, t \leq T\right)$ has a fair-price, indeed any EMM $\mathbb{Q}$ induces a no-arbitrage price given by $\mathbb{E}^{\mathbb{Q}}[\xi]$. Moreover, according to the definition of the major concrete model actually used in the financial arena, we also assume linearity and continuity assumptions. That's the modeler has access to the the price of European calls for any possible strike price, thus the function $f(y):=\mathbb{E}^{\mathbb{P}}\left[\left(S_{T}-y\right)^{+}\right]$is known for any strike price $y \in \mathbb{R}$, and the marginal distribution of $S_{T}$ is known. Hence, the set of admissible EMMs consists of all the EMM which are consistent with the aforementioned information, namely those EMM $\mathbb{Q}$ such that $\mathbb{Q} \circ\left(X_{0}\right)^{-1}=\delta_{S_{0}}=\mathbb{P} \circ\left(S_{0}\right)^{-1}$ and 
$\mathbb{Q} \circ\left(S_{T}\right)^{-1}=\mu=\mathbb{P} \circ\left(S_{T}\right)^{-1}$. We denote with $\mathbb{M}_{\mu}(S, \mathbb{P})$ the set of such measures. Therefore, the natural formulation of the upper bound problem for the no-arbitrage price associated to $\xi$ is given by $\sup \mathbb{E} \mathbb{Q}^{[\xi]}$ with optimization on $\mathbb{M}_{\mu}(S, \mathbb{P})$.

This problem is typically solved in literature ini terms of the Skorokhod Embedding problem (SEP). The latter framework can be exploited to study a derivative security $g\left(S_{s}, s \leq t\right)$ aiming to search for a suitable function $\lambda$, together with a martingale $M$, such that $\mathbb{E}\left[g\left(S_{s}, s \leq t\right)\right] \leq \lambda\left(S_{t}\right)+M_{t}$. In this way, for any stopping time $\tau$ such that $S_{\tau}$ distributed according to the law $\mu$, the following holds $\mathbb{E}\left[g\left(S_{s}, s \leq \tau\right)\right] \leq \int \lambda d \mu$, see, e.g., [13] and [14]. Nevertheless, latter method requires the payoffs to be invariant under time change, a restriction that we will eliminate in the present work. In particular, we directly solve the robust SP, hence obtaining a solution which provides $\lambda$ and $M$ as above. In order to develop the proposed technique we will mainly refere to [27] and [24] as main references.

It follows that the main aim of the present paper is to generalize the theory and the results achieved by Galichon, Labordere and Touzi in [12], to allow $S$ to be $\mathbb{P}$-semimartingale process. Latter result turns to be particularly useful for numerical implementations, as we will show, exploiting [12] and then relating the solution to the robust SP, to a singular stochastic control problem that can be approximated by a finite Monte Carlo differences scheme, see, e.g., [2], or exploiting Polynomial Chaos Expansion approaches, see, e.g., [10].

The work is organized as follows: in Section 2 we report the general setting, in Section 3 we state and prove the main results, while in Section 4 we provide the provide a possible computational scheme that will be written in terms of a no-arbitrage bound price problem.

\section{General Framework}

In what follows we consider an incomplete free-arbitrage market, allowing for static trading of European call options as well as the dynamic trading of the underlying $d$-dimensional risky asset denoted by $S=\left(S^{j}\right)_{1 \leq j \leq d}$, assumed to be a $d$-dimensional continuous $\mathbb{P}$-semimartingale (i.e. every $S^{j}$ is a continuous $\mathbb{P}$-semimartingale). Hence we assume that there exists a $\mathbb{P}$-Brownian motion $W$, a process $A$ with finite variation on compact sets, w.r.t.t, and a process $\alpha \in \mathscr{L}^{1}(W)$, such that the dynamic of the asset $S$ reads as follow

$$
S=S_{0}+\int_{0}^{t} A_{t} d u \int_{0}^{t} \alpha_{u} d W_{u}
$$


We recall that a self-financing portfolio $\Pi$ is defined as a pair $(x, H)$, where It consists $x \in \mathbb{R}_{+}$denotes the initial capital, while $H=\left(H^{j}\right)_{1 \leq j \leq d}$, with $H \in \mathscr{L}^{1}(S)$ and predictable, indicates the investment strategy by specifying the amount of each assets held.

For any strategy $H$ and initial capital $x$, the associated value process $X=$ $\left(X_{t}\right)_{0 \leq t \leq T}$ is determined by:

$$
X_{t}^{H}=x+\int_{0}^{t} H_{u} d S_{u} .
$$

For any initial capital $x \in \mathbb{R}_{+}$, we denote the set of admissible strategy by:

$$
\mathscr{H}(x):=\left\{H \in \mathscr{L}^{1}(S) \text { predictable } \mid X^{H} \geq 0\right\},
$$

and by the linearity assumption on the market, we have that $\mathscr{H}(x)=x \mathscr{H}(1)=$ $\left\{x_{0} H \mid H \in \mathscr{H}(1)\right\}$, indicating $\mathscr{H}(1)$ for short by $\mathscr{H}$. Moreover, adding the continuity assumption, we can assume that the marginal distribution $\mu$ of the asset $S$, at time $T$ as known, see [5].

In the present framework, the set of equivalent local martingale measures (ELMM) consists in those measures $\mathbb{Q} \sim \mathbb{P}$ under which $S$ is a local martingale. Following Tan and Touzi, see [28], we denote by $\mathbb{M}_{l}(S, \mathbb{P})=\mathbb{M}_{l}$ any ELMM $\mathbb{Q}$ such that $\mathbb{Q} \circ\left(S_{0}\right)^{-1}=\delta_{S_{0}}=\mathbb{P} \circ\left(S_{0}\right)^{-1}$, with $\mathbb{Q} \circ\left(S_{T}\right)^{-1}=\mu=\mathbb{P} \circ\left(S_{T}\right)^{-1}$. IN what follows we shall consider the case of $S$ to be locally bounded hence, following the idea of Kramkov and Schachermeyer, see [16, 17, 18], we have that $S$ is a local martingale under every $\mathbb{Q} \in \mathbb{M}_{l}$. By the free of arbitrage hypothesis, we have the existence of a measure of the previous type, e.g. applying the Girsanov-Meyer theorem, therefore we always assume $\mathbb{M}_{l} \neq \emptyset$. For any given $x \in \mathbb{R}_{+}$we refer to a derivative $\xi(x):=\xi$ by means of an $\mathcal{F}_{T}$-measurable random variable with $\mathbb{E}^{\mathbb{P}}[\xi]<\infty$, which is identify with an absolutely continuous function $\xi: \Omega_{x} \rightarrow \mathbb{R}$, where $\Omega_{x}:=\left\{\omega \in \mathcal{C}\left([0, T], \mathbb{R}_{+}^{d}\right) \mid \omega_{0}=x\right\}$.

Moreover, to avoid doubling strategies, for a given derivative $\xi$, and an initial capital $x \in \mathbb{R}$, we define the set of admissible portfolios as follows

$$
\begin{aligned}
\mathcal{H}(\xi, x):= & \left\{H \in \mathscr{H}(x) \mid X^{H} \text { is a } \mathbb{Q}\right. \text {-supermartingale : } \\
& \left.X_{T}^{H} \geq \xi \mathbb{Q} \text {-a.s. } \forall \mathbb{Q} \in \mathbb{M}_{l}\right\}
\end{aligned}
$$

Under the assumptions made so far, the superhedging problem (SP) is then represented by:

$$
U^{0}(\xi):=\inf \left\{x \in \mathbb{R}_{+} \mid \mathcal{H}(\xi, x) \neq \emptyset\right\},
$$


so that $U^{0}$ can be seen as the no-arbitrage lower bound on the market price of the derivative $\xi$ from the point of view of an investor who has access to a continuous time trading on the underlying securities whose prices follow the dynamics given by $S$. Analogous the SP consists in establishing a minimum cost for a suitable hedging portfolio whose marginal distribution at maturity $T$, is given by the law of $\xi$.

\section{Dual Formulation Problem}

In the present section we exploit the financial setting defined so far to provide a generalization of the dual formulation of the SP, as it has been given in [12]. In particular, the following theorem holds

Theorem 1 (Dual formulation of superhedging problem). Given a derivative $\xi$ such that

$$
\sup _{\mathbb{Q} \in \mathbb{M}_{l}} \mathbb{E}^{\mathbb{Q}}[\xi] \neq \pm \infty \quad \text { (well posedness of the } S P \text { problem) }
$$

the following equality holds:

$$
U^{0}(\xi) \equiv \sup _{\mathbb{Q} \in \mathbb{M}_{l}} \mathbb{E}^{\mathbb{Q}}[\xi]
$$

and there exist a predictable process $H \in \mathscr{L}^{1}(S)$, and a family of non-decreasing predictable processes $\left\{L_{t}^{\mathbb{Q}}\right\}_{t \in[0, T]}$, s.t. $\forall \mathbb{Q} \in \mathbb{M}_{l}, L_{0}^{\mathbb{Q}}=0$, and the following holds

$$
\xi=U^{0}(\xi)+\int_{0}^{T} H_{t} d S_{t}-L_{T}^{\mathbb{Q}} \quad \mathbb{Q} \text {-a.s. } .
$$

To prove Th 1, we first need to introduce some properties about the regular conditional probability distribution ( $\mathrm{rcpd}$ ), whose existence is derived by Stroock and Varadhan in [26]. In particular, we the notations introduced so far and following [12], we denote the rcpd of $\mathbb{P}$ w.r.t. a stopping time $\tau$, by $\mathbb{P}_{\tau}^{\omega}$, defined by means of the following properties:

- $\mathbb{P}_{\tau}^{\omega}$ is a probability measure on $\mathcal{F}_{T}$ for every $\omega \in \Omega$;

- the map $\omega \rightarrow \mathbb{P}_{\tau}^{\omega}(E)$ is $\mathcal{F}_{\tau}$-measurable for all $E \in \mathcal{F}_{T}$;

- for every bounded $\mathcal{F}_{T}$-measurable random variable $\xi, \mathbb{P}$-a.s. it holds:

$$
\mathbb{E}^{\mathbb{P}}\left[\xi \mid \mathcal{F}_{\tau}\right](\omega)=\mathbb{E}^{\mathbb{P} \omega}[\xi]
$$


- for all $\omega \in \Omega$ the following property holds:

$$
\mathbb{P}_{\tau}^{\omega}[\zeta \in \Omega \mid \zeta(s)=\omega(s), \text { for } 0 \leq s \leq \tau(\omega)]=1
$$

Then, we introduce the so called shifted framework, where, unless otherwise noted, the following definitions have to be understood for $t \in[0, T]$. In particular, the shifted canonical space is defined as

$$
\Omega^{t}:=\left\{\omega \in \mathscr{C}\left([t, T] ; \mathbb{R}^{d}\right) \mid \omega_{t}=0\right\}
$$

Consistently with the definition given along previous sections, we denote by $W^{t}$ the shifted canonical process on $\Omega^{t}$, whose associated shifted Wiener measure is denoted by $\mathbb{P}^{t}$, while $\mathcal{F}^{t}$ shall represent the shifted filtration generated by $W^{t}$ itself.

For $0 \leq s \leq t \leq T$ and $\omega^{s} \in \Omega^{s}$, we define:

- the shifted path $\omega^{t} \in \Omega^{t}$ as:

$$
\omega_{r}^{t}:=\omega_{r}^{s}-\omega_{t}^{s}, \text { for all } r \in[t, T],
$$

therefore, if we restrict to stationary processes, i.e. if we consider the canonical process, we have $\omega_{r}^{t} \equiv \omega_{r-t}^{s}$;

- the concatenation path $\omega^{s} \otimes_{t} \tilde{\omega} \in \Omega^{s}$ for some $\tilde{\omega} \in \Omega^{t}$ as:

$$
\left(\omega^{s} \otimes_{t} \tilde{\omega}\right)(r):=\omega_{r}^{s} \mathbb{I}_{[s, t)}(r)+\left(\omega_{t}^{s}+\tilde{\omega}_{r}\right) \mathbb{I}_{[t, T]}(r) \quad \text { for all } r \in[s, T]
$$

Note that $\left(\omega^{s} \otimes_{t} \tilde{\omega}\right)$ has again continuous paths. Indeed, the only discontinuity point could be the one in which the two different paths concatenate, but, exploiting the definition, it is easy to see that:

$$
\lim _{r \uparrow t}\left(\omega^{s} \otimes_{t} \tilde{\omega}\right)(r)=\lim _{r \uparrow t} \omega_{r}^{s} \mathbb{I}_{[s, t)}(r)=\omega_{t}^{s}=\lim _{r \downarrow t}\left(\omega_{t}^{s}+\tilde{\omega}_{r}\right) \mathbb{I}_{[t, T]}(r),
$$

since $\tilde{\omega} \in \Omega^{t}$.

Furthermore, by definition, we have $\left(\omega^{s} \otimes_{t} \tilde{\omega}\right)(t)=\omega_{t}^{s}$, we can conclude that $\left(\omega^{s} \otimes_{t} \tilde{\omega}\right)$ has continuous trajectories on the whole interval $[s, T]$.

- the shifted $\mathcal{F}_{T}^{t}$-measurable r.v. $\xi^{t, \omega}$ of some $\mathcal{F}_{T}^{s}$-measurable r.v. $\xi^{s}$ on $\Omega^{s}$ is defined as follows:

$$
\xi^{t, \omega}(\tilde{\omega}):=\xi^{s}\left(\omega^{s} \otimes_{t} \tilde{\omega}\right) \text {, for all } \tilde{\omega} \in \Omega^{t}
$$


Defining, for any $\mathcal{F}^{s}$-progressively measurable process $X^{s}$ on $[s, T]$, the shifted process on $[t, T]$, by $X^{t, \omega}(\tilde{\omega}):=X^{s}\left(\omega^{s} \otimes_{t} \tilde{\omega}\right)$, it is easy to see that the latter is $\mathcal{F}^{t}$-measurable.

Since $\mathbb{P}_{\tau}^{\omega}$ induces a probability measure $\mathbb{P}^{\tau, \omega}$ on $\mathcal{F}_{T}^{\tau}$, s.t. the $\mathbb{P}^{\tau, \omega}$-distribution of $W^{\tau}$ equals the $\mathbb{P}_{\tau}^{\omega}$-distribution of $\left\{W_{t}-W_{\tau}, t \in[\tau, T]\right\}$, or, equivalently the $\mathbb{P}^{\tau, \omega}$-distribution of $S^{\tau}$ is equal to the $\mathbb{P}_{\tau}^{\omega}$-distribution of $\left\{S_{t}-S_{\tau}, t \in[\tau, T]\right\}$, then a more intuitively way to interpret the rcpd for an $\mathcal{F}_{T}$-measurable r.v $\xi$, also in the particular case of a derivative, is given by the following identity:

$$
\mathbb{E}^{\mathbb{P}_{\tau}^{\omega}}[\xi]=\mathbb{E}^{\mathbb{P}^{\tau, \omega}}\left[\xi^{\tau, \omega}\right]
$$

Let us denote by $\mathbb{M}_{l}^{t}\left(S^{t}, \mathbb{P}^{t}\right):=\mathbb{M}_{l}^{t}$ the set of ELMM $\mathbb{Q}^{t}$ on $\Omega^{t}$ for the underlying shifted stock price process $S^{t}$ wrt $\mathbb{P}^{t}$, which satisfies $\mathbb{Q}^{t} \circ\left(S_{t}^{t}\right)^{-1}=\delta_{0}$ and $\mathbb{Q}^{t} \circ$ $\left(S_{T}^{t}\right)^{-1}=\mathbb{P}^{t} \circ\left(S_{T}^{t}\right)^{-1}$. Then, $\mathbb{Q} \in \mathbb{M}^{t}$ if and only if the shifted stock price process $S^{t}$ is a $\mathbb{Q}^{t}$-martingale, or a local $\mathbb{Q}^{t}$-martingale if $S$ is not bounded but only locally bounded on the time interval $[t, T]$.

Proof of the duality Theorem 1. Following [12], the dual result appearing in the statement is proven in two steps: first we show that $U^{0}(\xi) \geq \sup _{\mathbb{Q} \in \mathbb{M}_{l}}$, then, we exploit the rcpd properties to provide the inverse inequality.

first step : If there are not arbitrage opportunities, then, for any derivative $\xi$, we have $\mathbb{M}_{l}(\xi, \mathbb{P}) \neq \emptyset$, in particular $U^{0}(\xi)>-\infty$.

Let us consider $X_{0} \in \mathbb{R}_{+}$, s.t. there exists a strategy $H \in \mathcal{H}\left(\xi, X_{0}\right)$, $\mathbb{P}$ -a.s., for which it holds $X_{T}^{H} \geq \xi$. Then, by definition, the process $X^{H}$ is a $\mathbb{Q}$-local martingale and a $\mathbb{Q}$-supermartingale, for any $\mathbb{Q} \in \mathbb{M}_{l}$. Hence, from the arbitrariness on both $X_{0}$ and $\mathbb{Q}$, it follows that $X_{0} \geq \mathbb{E}^{\mathbb{Q}}[\xi]$, for any $\mathbb{Q} \in \mathbb{M}_{l}$. In particular the latter shows that

$$
U^{0}(\xi) \geq \sup _{\mathbb{Q} \in \mathbb{M}_{l}} \mathbb{E}^{\mathbb{Q}}[\xi]
$$

second step : In order to prove the inverse inequality, we start noticing that, by definition, $\xi \in U C\left(\Omega_{X_{0}}\right)$, then there exists the modulus of a continuous function $\rho$ such that, for all $t \in[0, T]$, the following holds:

$$
\left|\xi^{t, \omega}\left(\omega^{t}\right)-\xi^{t, \tilde{\omega}}\left(\omega^{t}\right)\right| \leq \rho\left(\|\omega-\tilde{\omega}\|_{t}\right),
$$

for any $\omega, \tilde{\omega} \in \Omega, \omega^{t} \in \Omega^{t}$, where

$$
\|\omega\|_{t}:=\sup _{s \in[0, t]}\left|\omega_{s}\right| .
$$


We now define a dynamic value process $V$, for all $(t, \omega) \in[0, T] \times \Omega$, as follows

$$
V_{t}(\omega):=\sup _{\mathbb{Q} \in \mathbb{M}_{l}^{t}} \mathbb{E}^{\mathbb{Q}_{t}^{\omega}}[\xi] .
$$

As noticed in [12], the uniform continuity property of $\xi$ entails that $\left\{V_{t}\right\}_{t \in[0, T]}$ is a right-continuous $\mathcal{F}_{T}$-adapted process, moreover, by the well posedness of the problem, we have:

$$
V_{t} \in \mathscr{L}^{1}(\mathbb{Q}) \text { for all } \mathbb{Q} \in \mathbb{M}_{l}
$$

Furthermore, by [25, Prop.4.7], we have that $V$ is actually a $\mathbb{Q}$-supermartingale. Thus, applying the Doob-Meyer decomposition, we can assume the existence of a pair process $\left(H^{\mathbb{Q}}, K^{\mathbb{Q}}\right)$, with $H^{\mathbb{Q}} \in \mathscr{L}^{1}(S)$ predictable and $K^{\mathbb{Q}}$ nondecreasing $\mathbb{Q}$-integrable, such that:

$$
V_{t}=V_{0}+\int_{0}^{t} H_{s}^{\mathbb{Q}} d S_{s}-K_{t}^{\mathbb{Q}} \quad \mathbb{Q} \text {-a.s. for all } t \in[0, T] .
$$

Moreover, since $V$ is a right-continuous $\mathbb{Q}$-semimartingale w.r.t. any $\mathbb{Q} \in \mathbb{M}_{l}$, we can use the properties of the $\mathscr{I}$ function introduced by Karandikar in [15] to aggregate the family processes $\left\{H_{t}^{\mathbb{Q}}, \mathbb{Q} \in \mathbb{M}_{l}\right\}$ into a process $\hat{H}$ defined on $[0, T] \times \Omega$ by the relation:

$$
d<V, S>_{t}=\hat{H}_{t} d<S>_{t} .
$$

Since the operator $\langle X, Y\rangle$ denotes the quadratic covariation process associated to the processes $X$ and $Y$, this relation has to be understood as $\hat{H}=H^{\mathbb{Q}}, d t \times d \mathbb{Q}$-a.s., and for all $\mathbb{Q} \in \mathbb{M}_{l}$.

Therefore, the previous relation can be written as:

$$
V_{t}=V_{0}+\int_{0}^{t} \hat{H}_{s} d S_{s}-K_{t}^{\mathbb{Q}} \quad \mathbb{Q} \text {-a.s. for all } \mathbb{Q} \in \mathbb{M}_{l}, t \in[0, T] .
$$

Moreover, taking $X_{0}:=V_{0}$, we have

- the process $\hat{H}_{t}:=X_{0}+\int_{0}^{t} \hat{H}_{s} d S_{s}$ is bounded from below by $V_{t}$, which is in turn bounded from below by $M_{t}^{\mathbb{Q}}:=\mathbb{E}_{t}^{\mathbb{Q}}[\xi]$. the latter being a local $\mathbb{Q}$-martingale since $\xi \in \mathscr{L}^{1}(\mathbb{Q})$. Moreover. this entails that $X^{\hat{H}}$ is a $\mathbb{Q}$-supermartingale, for all $\mathbb{Q} \in \mathbb{M}_{l}$;

- we also have that $X_{T}^{\hat{H}}=V_{T}+K_{T}^{\mathbb{Q}}=\xi+K_{T}^{\mathbb{Q}} \geq \xi$, $\mathbb{Q}$-a.s., for all $\mathbb{Q} \in \mathbb{M}_{l}$. 
In particular, from the definition of $U^{0}(\xi)$, we have that $V_{0} \geq U^{0}(\xi)$.

To conclude the proof, is enough to note that, as a consequence of the supermartingale property of $X^{\hat{H}}$ under every $\mathbb{Q} \in \mathbb{M}_{l}$, we have:

$$
V_{0}+\sup _{\mathbb{Q} \in \mathbb{M}_{l}} \mathbb{E}^{\mathbb{Q}}\left[-K_{T}^{\mathbb{Q}}\right] \geq \sup _{\mathbb{Q} \in \mathbb{M}_{l}} \mathbb{E}^{\mathbb{Q}}\left[X_{T}^{\hat{H}}-K_{T}^{\mathbb{Q}}\right]=\sup _{\mathbb{Q} \in \mathbb{M}_{l}} \mathbb{E}^{\mathbb{Q}}[\xi]=V_{0}
$$

Furthermore, since $K^{\mathbb{Q}}$ is nondecreasing, then $X^{\hat{H}}$ is a local $\mathbb{Q}$-martingale for all $\mathbb{Q} \in \mathbb{M}_{l}$ and the $K^{\mathbb{Q}}$ satisfies the minimal condition

$$
\inf _{\mathbb{Q} \in \mathbb{M}_{l}} \mathbb{E}^{\mathbb{Q}}\left[K_{T}^{\mathbb{Q}}\right]=0
$$

\section{Concrete Hedging of Vanilla Options}

In what follows, we are going to exploit the results stated along previous sections, to analyse hedging strategies for the concrete treatment of Vanilla type options when the related underlying asset is defined as a semimartingale, our main aim being to provide a method suitable for numerical applications. Our financial setting is characterized by both linearity and continuity assumptions, moreover, see [5], we also assume that the marginal distribution $\mu$ of the aforementioned (semimartingale) underlying asset $S$, at time $T$, is given and we denote by $\mathcal{M}\left(\mathbb{R}_{+}^{d}\right)$ the set of probability measure on $\mathbb{R}_{+}^{d}$. Previous assumptions concerning $\lambda \in \mathscr{L}^{1}(\mu)$, allow us to write the $T$-maturity European derivative defined by the payoff $\lambda\left(S_{T}\right)$, by mean of an unambiguous, no-arbitrage price given by $\mu(\lambda)=\int \lambda d \mu$, which can be perfectly replicated, see [6]. Furthermore, given an initial spot price $S_{0}$ for $S$, the probability $\mu$ has to satisfy $\int_{\mathbb{R}^{d}} x d \mu(x)=S_{0}$, namely the fair price in the particular case $\lambda=\mathbb{I}_{\mathbb{R}_{+}}$. Following [12], we strength the hypothesis on the payoff, defining

$$
\Lambda^{\mu}:=\left\{\lambda \in \mathscr{L}^{1}(\mu) \mid \sup _{\mathbb{Q} \in \mathbb{M}_{l}} \mathbb{E}^{\mathbb{Q}}\left[\lambda\left(S_{T}\right)\right]<\infty\right\}
$$

and the space restriction for the payoff, related to the $\mathcal{F}_{T}$-measurable r.v. $\xi$, by

$$
\Lambda_{U C}^{\mu}:=\Lambda^{\mu} \cap U C(\mathbb{R}),
$$

where $U C(\mathbb{R})$ is the set of uniformly continuous maps $\mathbb{R} \rightarrow \mathbb{R}$.

For every pair $\xi$ and $\lambda \in \Lambda_{U C}^{\mu}$, as before, one defines the residual security of $\xi$ as $\xi^{\lambda}:=\xi-\lambda\left(S_{T}\right)$. Recalling that

$$
\begin{gathered}
\mathcal{H}(\xi, x):=\left\{H \in \mathscr{H}(x) \mid X^{H} \text { is a } \mathbb{Q}\right. \text {-supermartingale : } \\
\left.X_{T}^{H} \geq \xi \mathbb{Q} \text {-a.s. } \forall \mathbb{Q} \in \mathbb{M}_{l}\right\},
\end{gathered}
$$


the problem of no-arbitrage upper-bound of the $\xi$-payof $f$, provided we are superhedging $\nu(\xi, \lambda):=\xi+\mu(\lambda)-\lambda\left(S_{T}\right)$, reads as follows

$$
U^{\mu}(\xi):=\inf \left\{x \mid \exists \lambda \in \Lambda_{U C}^{\mu}: \mathcal{H}(\nu(\xi, \lambda), x) \neq \emptyset\right\}
$$

where

$$
\bar{X}_{T}^{H, \lambda}=X_{T}^{H}-\mu(\lambda)+\lambda\left(S_{T}\right) .
$$

Therefore, we are minimizing the cost of (super)hedging some derivative $\xi$ through a portfolio $H$, requiring that, at the maturity time $T$, our portfolio value process is at least equal to the one associated to the derivative, w.r.t. any equivalent local martingale measure. Actually, it refers to a portfolio value of a self-financing strategy, with continuous trading $H$ in the primitive security, and static trading $\lambda$ in the $T$-maturity, keeping consideration of the associated no arbitrage price $\mu(\lambda)$ for the option with such a payoff.

The following result, mainly based on the analogous provided in [12], is a direct consequence of the duality theorem 1

Theorem 2. Let $\mu \in \mathcal{M}\left(\mathbb{R}_{+}\right)$and $\xi \in U C\left(\Omega_{x}\right)$ such that $\sup _{\mathbb{Q} \in \mathbb{M}_{l}} \mathbb{E}^{\mathbb{Q}}[\xi]<$ $\infty$, then

$$
U^{\mu}(\xi)=\inf _{\lambda \in \Lambda_{U C}^{\mu}} \sup _{\mathbb{Q} \in \mathbb{M}}\left\{\mu(\lambda)+\mathbb{E}^{\mathbb{Q}}\left[\xi-\lambda\left(S_{T}\right)\right]\right\}
$$

Proof. The result straight follows from the proof of Th. 1, since we can write $U^{\mu}(\xi)=\inf _{\lambda \in \Lambda_{U C}^{\mu}} U^{0}(\nu)$. We refer the interested reader to [12] for more details.

We underline that the latter dual formulation gives access to the optimal hedging strategy $\lambda^{*}$, namely the optimal $T$-maturity vanilla profile, and the worst case model $\mathbb{Q}^{*}$ corresponding to the upper bound. The optimal dynamic hedging strategy in the underlying asset is then obtained by mean of the optimal residual security $\xi-\lambda^{*}\left(S_{T}\right)$ and concrete numerical approximations can be based on it as done, e.g., in $[4,19]$. It is worth to mention that, in order, to derive accurate numerical results a deeper study has to be developed concerning the value of the relevant parameters of the particular financial model we are interested in. In particular, also in the case of plain vanilla options, phenomena as, e.g., the volatility smile, cannot be avoided. Therefore a fine tuning of both the drift and the volatility variables, see, e.g., [10], should be conducted before any numerical scheme implementations, no matter if they are of the standard Monte Carlo type, see, e.g., [2], or if they are based on faster, even if ad hoc, computational techniques as done, e.g., in [3]. 


\section{Conclusions and Possible Developments}

In the present paper we have addressed the superhedging problem in a freearbitrage, incomplete market, avoiding the classical restriction of dealing only with derivatives having time invariant payoff, which is customary to assume in order to exploit the Skorokhod Embedding Problem (SEP) approach. In particular we have exploited the Monge-Kantorovich (MK) dual approach that turns the starting problem into a problem of stochastic optimization with linear constrain without requiring any time invariant property. Therefore, we have transformed the initial superhedging dual problem for a given derivative into the quest for its lower bound fair price. In doing that, we have generalized the results provided in [12], to consider assets whose dynamic admits continuous semimartingale properties. We also provide an alternative dual formulation of the initial problem which can be used for numerical approximations, also pointing out how related computational and accuracy difficulties can be taken into account. We would like to underline that the approach developed in this paper can be generalised to consider more complex financial derivatives structure. In particular, we aim at extending the results here reported mainly towards two directions: first to consider the case of assets whose dynamics are subjected to jump components by exploiting approximation results developed in [21], and then to take into account derivatives characterized by backward components hence covering the approach developed, e.g., in $[7,9,11]$, also with respect to stochastic optimization problems, as treated in, e.g., [22], with techniques as those developed in [1] which allow to treat non linear drift terms.

\section{References}

[1] V. Barbu, F. Cordoni, and L. Di Persio, Optimal control of stochastic FitzHugh-Nagumo equation, International Journal of Control, vol. 89, no. 4, pp. 746756, (2016), DOI: 10.1080/00207179.2015.1096023

[2] Phelim P. Boyle, Options: A Monte Carlo approach, Journal of Financial Economics 4 (1977), 1977, DOI: 10.1016/0304-405X(77)90005-8.

[3] M. Bonollo, L. Di Persio and G. Pellegrini, Polynomial chaos expansion approach to interest rate models, Journal of Probability and Statistics Vol. 2015, Art. NO. 369053, (2015), http://dx.doi.org/10.1155/2015/369053

[4] J. Frederic Bonnans, Xiaolu Tan, A model-free no-arbitrage price bound for variance options, Applied Mathematics and Optimization, Springer Verlag (Germany), 68 No.1 (2013), 43-73.

[5] D. T. Breeden, R. H. Litzenberger, Prices of state-contingent claims implicit in options price, Journal of Businness No. 51 (1978), 621-651 
[6] P. Carr, A. Chou, Breaking barriers Risk, No. 10 (1997), 139-145

[7] F. Cordoni and L. Di Persio, Backward stochastic differential equations approach to hedging, option pricing, and insurance problems, International Journal of Stochastic Analysis, vol. 2014, Article ID 152389, (2014) http://dx.doi.org/10.1155/2014/152389

[8] F. Cordoni and L. Di Persio, Invariant measure for the Vasicek interest rate model in the Heath-Jarrow-Morton-Musiela framework, Infinite Dimensional Analysis Quantum Probability and Related Topics, vol. 18, no. 3, (2015) http://dx.doi.org/10.1142/S0219025715500228

[9] F. Cordoni and L. Di Persio, A BSDE with Delayed Generator Approach to Pricing under Counterparty Risk and Collateralization, International Journal of Stochastic Analysis, vol. 2016, Article ID 1059303, (2016), doi:10.1155/2016/1059303

[10] L. Di Persio and M. Frigo, Gibbs sampling approach to regime switching analysis of financial time series, Journal of Computational and Applied Mathematics, vol. 300, (2016) doi:10.1016/j.cam.2015.12.010

[11] El Karoui, Peng, Quenez, Backward Stochastic Differential Equation in Finance, Mathematical Finance, Vol. 7, No. 1 (January 1997), 171, DOI: 10.1111/1467-9965.00022.

[12] A. Galichon, P. Henry-Labordere, T. Touzi, A stochastic control approach to no-arbitrage bounds given marginals, with an application to lookback options, The annals of applied probability, 24 No. 1 (2014), 312-336, doi:10.1214/13-AAP925

[13] D. Hobson, Robust hedging of the lookback options, textitStochastic Finance, No.2 (1998), 329-347, doi:10.1007/s007800050044

[14] D. Hobson, The Skorokhod embedding problem and model- independent bound for option prices, Lecture Notes in Mathematics, Springer, No. 2003 (2009), 267-318

[15] R. L. Karandikar, On pathwise stochastic integration, textitStochastic processes Application, No. 57 (1995), 11-18, doi:10.1016/0304-4149(95)00002-O

[16] D. Kramkov, W. Schachermayer, The asymptotic elasticity of utility functions and optimal investment in incomplete markets, textitThe annals of applied probability, textbf9, No. 3 (1999), 904-950, doi:10.1214/aoap/1029962818

[17] D. Kramkov, W. Schachermayer, Necessary and sufficient condition in the problem of optimal investment in incomplete markets, The annals of applied probability, 13 No. 4 (2003), 1504-1516, doi:10.1214/aoap/1069786508

[18] D. Kramkov, W. Schachermayer, J. Hugonnier, On utility-based pricing of contingent claims on incomplete markets, Mathematical finance, 15 No. 2 (2005), 203-212

[19] K. Larsen, O. Mostovyi, G. Zitkovic, An expansion in the model space in the context of utility maximization, arxiv (2014), arXiv:1410.0946

[20] R. Liptser, A. Shiryayev Nikolayevich, Theory of Martingales, Springer, (1989)

[21] C. Marinelli, L. Di Persio, and G. Ziglio, Approximation and convergence of solutions to semilinear stochastic evolution equations with jumps, Journal of Functional Analysis, vol. 264, no. 12, (2013) DOI: 10.1016/j.jfa.2013.02.020

[22] E. Pardoux, S. Peng, Adapted Solutions of Backward stochastic differential equation, Systems and Control Letters 14 (1990), 55-61, DOI: 10.1016/0167-6911(90)90082-6.

[23] Philip E. Protter, Stochastic integration and differential equations, Springer - Probability Theory and Stochastic Processes, (2004) 
[24] S. T. Rachev, L. Ruschendorf, Mass transportation problem, Springer - Probability and its application (1998)

[25] H. M. Soner, N. Touzi, J. Zhang, Dual formulation of second order target problems, Annals of Applied Probability No. 23 (2013), 308-347, doi:10.1214/12-AAP844

[26] D. W. Stroock, S. R. S. Varadhan, Multidimensional diffusion processes Fundamental Principles of Mathematical Sciences No. 233 (1979)

[27] C. Villani, Optimal transport, old and new, Springer (2008)

[28] X. Tan, N. Touzi, Optimal transportation under controlled stochastic dynamics, The annals of probability 41 No. 5 (2013), 2301-3240, doi:10.1214/12-AOP797 
